Short Note

\title{
4-(\{4-[(2E)-3-(2,5-Dimethoxyphenyl)prop-2- enoyl]phenyl\}amino)-4-oxobutanoic Acid
}

\author{
Hery Suwito *, Kautsar Ul Haq, Nia Nur Dinia Rahmah, Alfinda Novi Kristanti and \\ Ni Nyoman Tri Puspaningsih
}

Department of Chemistry, Faculty of Science and Technology, Airlangga University, Surabaya 60115, Indonesia; kautsar_ulhaq@yahoo.co.id (K.U.H.); nia.nurdinia.rahmah88@gmail.com (N.N.D.R.);

krisnosuwono@yahoo.com (A.N.K.); nyomantri@yahoo.com (N.N.T.P.)

* Correspondence: herys08032002@yahoo.com; Tel: +62-31-5922-427

Academic Editor: Norbert Haider

Received: 14 March 2017; Accepted: 29 March 2017; Published: 5 April 2017

\begin{abstract}
A dimethoxy amide chalcone has been synthesized in a two-step reaction. First, an amine chalcone was synthesized by the reaction of 4'-aminoacetophenone and 2,5-dimethoxybenzaldehyde using $40 \% \mathrm{NaOH}$ solution as a catalyst in ethanol, and then followed by amidation through the reaction of the formed chalcone and succinic anhydride. The structure of the target compound was established by FTIR, HR-MS, ${ }^{1} \mathrm{H}$ - and ${ }^{13} \mathrm{C}-\mathrm{NMR}$, and 2D-NMR spectral analysis.
\end{abstract}

Keywords: amino chalcones; amide chalcones; succinic anhydride

\section{Introduction}

Diaryl- $\alpha, \beta$-unsaturated ketones are the biogenic precursor in flavonoid biosynthesis [1] and are known as chalcones. Due to their wide spectrum of pharmacological properties, such as antioxidant [2], antihepatotoxic [3], neuroprotective [4], antibacterial [5-8], inhibitor of topoisomerase I [9], antimalarial [10,11], and anticancer [12], chalcones attract many researchers to develop efficient synthetic methods and to gain various structural variations of chalcones unavailable in nature. In general, chalcones are synthesized by Claisen-Schmidt condensation.

Previously we have reported the antimicrobial activity of a series of methoxy amino chalcones $[13,14]$. In order to enhance their efficacy by increasing their solubility and slow release, we converted the basic amino chalcones into amide derivatives through a reaction with succinic anhydride. Herein we report a new amide methoxy chalcone prepared from a methoxy amino chalcone and succinic anhydride.

\section{Results}

The title compound was synthesized in a two-step reaction. The first step was the synthesis of a methoxy amino chalcone (1) employing the Claisen-Schmidt reaction, then followed by the amidation of (1) through the reaction of (1) with succinic anhydride in ethanol using pyridine as a catalyst, as shown in Scheme 1. 
<smiles>COc1ccc(OC)c(/C=C/C(=O)c2ccc(NC(=O)COc3cc(OC)cc(C(=O)[PH2+])c3OC)cc2)c1</smiles>

(1)<smiles>COc1ccc(OC)c(/C=C/C(=O)c2ccc(NC(=O)CCC(=O)O)cc2)c1</smiles>

Scheme 1. Synthesis pathway of the target compound.

Firstly, the purity of the product was analyzed by determining its melting point and thin layer chromatography. The structure of the product was then characterized based on spectroscopic evidence and the results are displayed below. The product is assumed to exist in the $E$ configuration, since the ${ }^{1} \mathrm{H}-\mathrm{NMR}$ spectrum of the olefinic protons showed a coupling constant of $15.7 \mathrm{~Hz}$ indicative of the $E$ configuration. The structure of the title compound and its HMBC correlations is displayed in Figure 1, whereas the chemical shifts and its HMBC correlations is tabulated in Table 1. The complete spectra are attached in supplementary materials.

(E)-4-((3-(3-(2,5-Dimethoxyphenyl)acryloyl)phenyl)amino)-4-oxobutanoic acid (2): orange solid (266 mg; $75 \%$ ), m.p. $188-190{ }^{\circ} \mathrm{C}, R_{\mathrm{f}}=0.61$ (ethanol), HR-MS $[\mathrm{M}+\mathrm{H}]^{+}$calculated for $\mathrm{C}_{21} \mathrm{H}_{21} \mathrm{NO}_{6} 384.1447$, found 384.1446; IR (KBr, cm ${ }^{-1}$ ) 3448 (br, -OH carboxylic), 3340 (str, -NH- amide), 1720 (str, C=O aliphatic carboxylic acid), 1697 (str, $\mathrm{C}=\mathrm{O}$ amide), 1639 ( $\mathrm{C}=\mathrm{O}$ conjugated), 1593 (str, $\mathrm{C}=\mathrm{C}$ conjugated), and $1261\left(\mathrm{C}_{\text {alkyl }}-\mathrm{O}-\mathrm{C}_{\text {aryl }}\right) ;{ }^{1} \mathrm{H}-\mathrm{NMR}\left(400 \mathrm{MHz}, \mathrm{DMSO}-d_{6}\right) \delta(\mathrm{ppm}) 12.15(\mathrm{~s}, 1 \mathrm{H}), 10.32(\mathrm{~s}, 1 \mathrm{H})$, $8.10(\mathrm{~d}, J=8.7 \mathrm{~Hz}, 2 \mathrm{H}), 7.97(\mathrm{~d}, J=15.7 \mathrm{~Hz}, 1 \mathrm{H}), 7.86(\mathrm{~d}, J=15.7 \mathrm{~Hz}, 1 \mathrm{H}), 7.73(\mathrm{~d}, J=8.7 \mathrm{~Hz}$, $2 \mathrm{H}), 7.51(\mathrm{~d}, J=2.3 \mathrm{~Hz}, 1 \mathrm{H}), 7.08-6.92(\mathrm{~m}, 2 \mathrm{H}), 3.80(\mathrm{~s}, 3 \mathrm{H}), 3.76(\mathrm{~s}, 3 \mathrm{H}), 2.58(\mathrm{t}, J=6.2 \mathrm{~Hz}, 2 \mathrm{H})$, $2.50(\mathrm{t}, J=6.0 \mathrm{~Hz}, 2 \mathrm{H}) .{ }^{13} \mathrm{C}-\mathrm{NMR}\left(101 \mathrm{MHz}, \mathrm{DMSO}-d_{6}\right) \delta(\mathrm{ppm}) 188.05,174.33,171.35,153.77,153.16$, $144.19,138.06,132.64,130.46,124.08,122.36,118.73,118.57,113.53,112.93,56.66,56.22,31.71,29.13$.<smiles>COc1ccc(OC)c(/C=C/C(=O)c2ccc(NC(=O)CCC(=O)O)cc2)c1</smiles>

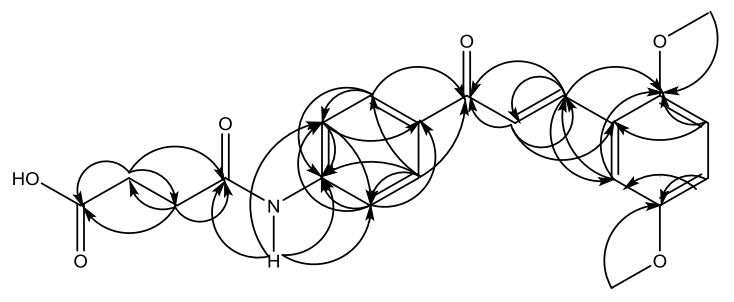

Figure 1. Structure, numbering and HMBC correlations of the title compound.

The analysis of the correlation spectrum (2D NMR; HMBC) is tabulated in the Table 1 below.

Table 1. ${ }^{1} \mathrm{H},{ }^{13} \mathrm{C}$ chemical shifts and HMBC correlations of the title compound.

\begin{tabular}{ccc}
\hline Atom & Chemical Shift (ppm) & HMBC \\
\hline $1 \mathrm{C}$ & 153.16 & \\
$1^{\prime} \mathrm{C}$ & 56.66 & \\
$\mathrm{H}_{3}$ & 3.80 & 1 \\
$2 \mathrm{C}$ & 113.53 & \\
$\mathrm{H}$ & 6.99 & 1,6 \\
\hline
\end{tabular}


Table 1. Cont

\begin{tabular}{|c|c|c|}
\hline Atom & Chemical Shift (ppm) & НМВС \\
\hline $3 \mathrm{C}$ & 118.57 & \\
\hline $\mathrm{H}$ & 6.99 & 4,5 \\
\hline $4 \mathrm{C}$ & 153.77 & \\
\hline $4^{\prime} \mathrm{C}$ & 56.22 & \\
\hline $\mathrm{H}_{3}$ & 3.76 & 4 \\
\hline $5 \mathrm{C}$ & 112.93 & \\
\hline $\mathrm{H}$ & 7.51 & $7,3,1$ \\
\hline $6 \mathrm{C}$ & 124.08 & \\
\hline $7 \mathrm{C}$ & 138.06 & \\
\hline $\mathrm{H}$ & 7.97 & $9,5,1,8$ \\
\hline $8 \mathrm{C}$ & 122.36 & \\
\hline $\mathrm{H}$ & 7.86 & $9,7,6$ \\
\hline $9 \mathrm{C}$ & 188.05 & \\
\hline $10 \mathrm{C}$ & 132.64 & \\
\hline $11 \mathrm{C}$ & 130.46 & \\
\hline $\mathrm{H}$ & 8.10 & $9,12,15,13$ \\
\hline $12 \mathrm{C}$ & 118.73 & \\
\hline $\mathrm{H}$ & 7.73 & $14,10,13$ \\
\hline $13 \mathrm{C}$ & 144.19 & \\
\hline $14 \mathrm{C}$ & 118.73 & \\
\hline $\mathrm{H}$ & 7.73 & $12,10,13$ \\
\hline $15 \mathrm{C}$ & 130.46 & \\
\hline $\mathrm{H}$ & 8.10 & $9,14,11,13$ \\
\hline $16 \mathrm{H}$ & 10.32 & $12,14,13,17$ \\
\hline $17 \mathrm{C}$ & 171.35 & \\
\hline $18 \mathrm{C}$ & 29.13 & \\
\hline $\mathrm{H}_{2}$ & 2.50 & $19,17,20$ \\
\hline $19 \mathrm{C}$ & 31.71 & \\
\hline $\mathrm{H}_{2}$ & 2.58 & $18,17,20$ \\
\hline $20 \mathrm{C}$ & 174.33 & \\
\hline $21 \mathrm{H}$ & 12.15 & \\
\hline
\end{tabular}

\section{Materials and Methods}

\subsection{General}

All reagents and solvents (E.Merck (Darmstadt, Germany) or Sigma Aldrich (St. Louis, MO, USA)) were used without further purification. Reaction progress was monitored by TLC on silica gel $\mathrm{GF}_{254}$ aluminum sheets $(0.25 \mathrm{~mm})$ using various developing system. Spots were detected under UV light $(\lambda 254 \mathrm{~nm})$. Melting point was measured by Thermo Scientific Fisher-Johns Melting Point Apparatus 220 VAC (Waltham, MA, USA) and uncorrected. FTIR spectrum was recorded in KBr pellet on FTIR spectrophotometer Shimadzu 84005 series (Kyoto, Japan). Mass spectrum was recorded on HR mass spectrometer Waters LCT Premier XE (Santa Clara, CA, USA). NMR spectrum $\left({ }^{1} \mathrm{H}-,{ }^{13} \mathrm{C}-\mathrm{NMR}\right.$, and HMBC) was recorded using JEOL 400 ECA spectrometer (Tokyo, Japan) with DMSO- $d_{6}$ as solvent and internal standard.

\subsection{Preparation of the Title Compound (2)}

The amino methoxy chalcone (1) was synthesized according to the protocol as described previously [2]. The title compound was synthesized as followed: $1 \mathrm{mmol}$ succinic anhydride was dissolved in $5 \mathrm{~mL}$ DCM, then three drops of pyridine was added. The mixture was stirred at $40{ }^{\circ} \mathrm{C}$ for $10 \mathrm{~min}$. Then $1 \mathrm{mmol}$ of chalcone (1) in $2 \mathrm{~mL}$ DCM was added drop-wise, stirred overnight at room temperature. The precipitate was then filtered off and re-crystallized from ethanol.

\section{Conclusions}

We have demonstrated the synthesis of a methoxy amide chalcone derivative through the Claisen-Schmidt reaction, followed by amidation. 
Supplementary Materials: FTIR, HRMS, ${ }^{1} \mathrm{H}-\mathrm{NMR},{ }^{13} \mathrm{C}-\mathrm{NMR}, \mathrm{HMBC}$ spectra of the synthesized compound are available online.

Acknowledgments: The study was funded by The Ministry of Research and Higher Education of Indonesia under PUPT research grant 2016.

Author Contributions: H.S. brought out the idea, managed the research and wrote the paper. K.U.H. and A.N.K. analyzed the spectral data. N.N.D.R. performed the synthesis, while N.N.T.P. corrected the draft. All the authors have read the draft.

Conflicts of Interest: The authors declare no conflict of interest.

\section{References}

1. Lin, M.; Zhou, Y.; Flavin, M.T.; Zhou, L.; Nie, W.; Chen, F. Chalcones and flavonoids as anti-tuberculosis agents. Bioorg. Med. Chem. 2002, 10, 2795-2782. [CrossRef]

2. Kim, B.T.; Chun, J.C.; Hwang, K.J. Synthesis of dihydroxylated chalcone derivatives with diverse substitution pattern and their radical scavenging ability toward DPPH free radicals. Bull. Korean Chem. Soc. 2008, 29, 1125-1130. [CrossRef]

3. Khan, S.A.; Ahmed, B.; Alam, T. Synthesis and hepatotoxic activity of some new chalcones containing 1,4-dioxane ring system. Pak. J. Pharm. Sci. 2006, 19, 290-294. [PubMed]

4. Jung, J.-C.; Jang, S.; Lee, Y.; Min, D.; Lim, E.; Jung, H.; Oh, M.; Oh, S.; Jumg, M. Efficient synthesis and neuroprotective effect of substituted 1,3-diphenyl-2-propen-1-ones. J. Med. Chem. 2008, 51, 4054-4058. [CrossRef] [PubMed]

5. Prasad, Y.R.; Kumar, P.R.; Smiles, D.J.; Babu, P.A. QSAR studies on chalcone derivatives as antibacterial agents against Bacillus pumilis. Arkivoc 2008, 11, 266-276.

6. Sharma, M.; Chaturvedi, V.; Manju, Y.K.; Bhatnagar, S.; Srivastava, K.; Puri, S.K.; Chauhan, P.M.S. Substituted quinolinyl chalcones and quinolinyl pyrimidines as a new class of anti-infective agents. Eur. J. Med. Chem. 2009, 44, 2081-2091. [CrossRef] [PubMed]

7. Asiri, A.M.; Khan, S.A. Synthesis and anti-bacterial activities of a bis-chalcone derived from thiophene and its bis-cyclized products. Molecules 2011, 16, 523-531. [CrossRef] [PubMed]

8. Sivakumar, P.M.; Seenivasan, S.P.; Kumar, V.; Doble, M. Synthesis, antimycobacterial activity evaluation, and QSAR studies of chalcone derivatives. Bioorg. Med. Chem. Lett. 2007, 17, 1695-1700. [CrossRef] [PubMed]

9. Yoon, G.; Kang, B.Y.; Cheon, S.H. Topoisomerase I inhibition and cytotoxicity of lichochalcones A and E from Glycyrrhiza inflate. Arch. Pharm. Res. 2007, 30, 313-316. [CrossRef] [PubMed]

10. Dave, S.S.; Ghatole, A.M.; Rahatgaonkar, A.M.; Chorghade, M.S.; Chauhan, P.M.S.; Srivastava, K. Experimental and computational evaluation of new quinolinyl chalcones as potent antiplasmodial agents. Indian J. Chem. 2009, 48, 1780-1793.

11. Suwito, H.; Pudjiastuti, P.; Fanani, M.Z.; Kimata-Ariga, Y.; Katahira, R.; Kawakami, T.; Fujiwara, T.; Hase, T.; Sirat, H.M.; Puspaningsih, N.N.T. Design and synthesis of chalcone derivatives as inhibitors of the ferredoxin-Ferredoxin-NADP+ reductase interaction of plasmodium falciparum: Pursuing new antimalarial agents. Molecules 2014, 19, 21473-21488. [CrossRef] [PubMed]

12. Ferrer, R.; Lobo, G.; Gamboa, N.; Rodrigues, J.; Abramjuk, C.; Jung, K.; Lein, M.; Charris, J.E. Synthesis of [(7-chaloroquinolin-4-yl)amino]chalcones: Potential antimalarial and anticancer agents. Sci. Pharm. 2009, 77, 725-741.

13. Suwito, H.; Jumina; Mustofa; Ni'matuzahroh; Puspaningsih, N.N.T. Anticancer and antimicrobial activity of methoxy amino chalcone derivatives. Der Pharma Chem. 2015, 7, 89-94.

14. Suwito, H.; Kristanti, A.N.; Hayati, S.; Dewi, S.R.; Amalina, I.; Puspaningsih, N.N.T. Antimicrobial activities and in silico analysis of methoxy amino chalcone derivatives. Procedia Chem. 2016, 18, 103-111. [CrossRef]

(C) 2017 by the authors. Licensee MDPI, Basel, Switzerland. This article is an open access article distributed under the terms and conditions of the Creative Commons Attribution (CC BY) license (http:/ / creativecommons.org/licenses/by/4.0/). 